\title{
Um modelo dinâmico de macroeconomia aberta com metas de inflação, conflito distributivo e equilíbrio na conta corrente
}

\author{
Carlos Eduardo Drumond ${ }^{* *}$ \\ Gabriel Porcile ${ }^{* * *}$
}

\begin{abstract}
Resumo
O artigo discute, sob uma perspectiva pós-keynesiana, o impacto do regime de metas de inflação sobre o crescimento, a distribuição e a estabilidade em uma economia aberta. O modelo combina a barganha salarial, as variações na capacidade utilizada e o equilíbrio em conta corrente para mostrar que a política monetária tem um impacto real sobre o crescimento e o emprego no longo prazo - i.e. existe um trade-off entre inflação e crescimento. São analisadas as implicações de uma regra de política monetária que leva em conta o equilíbrio em conta corrente. Mostra-se que esta regra poderia ser importante para sustentar a estabilidade no longo prazo, na medida em que evita a possibilidade de aumentos explosivos no estoque da dívida externa.
\end{abstract}

Palavras chave: Metas de inflação; Política monetária; Economia aberta; Economia pós-keynesiana; Conflito distributivo.

\section{Abstract \\ A dynamic model of open macroeconomics with inflation targets, conflicting claims and current account balance}

The paper discusses, from a Post-Keynesian perspective, the impacts of an inflation target regime on growth, distribution and stability in an open economy. The model combines a conflicting claims theory of inflation, changes in the rate of capacity utilization and equilibrium in the external sector to show that in the long run monetary policy has a real impact on growth and employment there exists a trade-off between the inflation rate and the growth rate. A monetary rule that takes into consideration equilibrium in current account is analyzed. It is shown that this rule can contribute to stability in the long run, to the extent that it hinders the possibility of explosive growth in the stock of external debt.

Keywords: Inflation targets; Monetary policy; Open economy; Post-Keynesian economics; Conflicting claims.

JEL E20, E50, F41.

* Trabalho recebido em 8 de abril de 2010 e aprovado em 12 de setembro de 2012.

** Professor do Departamento de Economia da Universidade Estadual de Santa Cruz (UESC) e Doutorando do Programa de Pós-Graduação em Desenvolvimento Econômico (UFPR), Curitiba, PR, Brasil. E-mail: carloseduardo.drumond@yahoo.com.br.

*** Professor Associado do Departamento de Economia da Universidade Federal do Paraná (UFPR) e Oficial de Assuntos Econômicos da Cepal, Curitiba, PR, Brasil. E-mail: porcile@ufpr.br. 


\section{Introdução}

Nas últimas décadas emergiu uma espécie de consenso na macroeconomia do mainstream cujos impactos têm sido sentidos tanto no campo acadêmico, quanto na prática da condução das políticas macroeconômicas. O escopo geral deste novo consenso se assenta na utilização de uma curva de Phillips novokeynesiana, numa regra de condução de política monetária e no uso de metas de inflação.

Por detrás deste consenso existem supostos teóricos sujeitos à crítica póskeynesiana, em especial a noção de taxa natural de crescimento do produto e de juros, que conduzem à neutralidade da moeda no longo prazo. Recentemente, uma série de modelos pós-keynesianos têm sido desenvolvidos na tentativa de propor uma estrutura mais realista para a condução da política macroeconômica. Um ponto importante em modelos como os apresentados por Setterfield (2006), Lima e Setterfield (2008) e Oreiro e Rocha (2008) é que eles advogam a possibilidade de compatibilizar a economia pós-keynesiana com o uso de metas de inflação, com a ressalva de que isto só é possível dentro de uma estrutura teórica muito distinta à dos modelos ortodoxos. O presente texto se localiza nessa linha de trabalhos, com foco num tema relativamente menos discutido até o momento. A maior parte dos textos citados se concentra em economias fechadas, enquanto que neste trabalho exploram-se as possibilidades de avançar num modelo pós-keynesiano com metas de inflação em economia aberta. Embora se deva reconhecer a importância dos modelos de macroeconomia fechada para entender alguns aspectos chave da dinâmica macroeconômica, em economias em desenvolvimento fortemente integradas ao comércio internacional e aos fluxos de capital, os modelos de economia aberta são particularmente necessários.

Assim, o objetivo deste trabalho é desenvolver um modelo dinâmico para macroeconomia aberta que inclua na curva de Phillips a barganha salarial, o conflito distributivo e a taxa de câmbio, assim como a proposição de uma regra de condução de política monetária que leve em conta o nível de utilização da capacidade instalada.

Três são as contribuições específicas do trabalho. Em primeiro lugar é mostrado que no longo prazo existe um trade-off entre inflação e crescimento e que a regra monetária utilizada pode ter efeitos reais sobre a economia, como sugerido pela tradição teórica keynesiana. Em segundo lugar, argumenta-se que em economias em desenvolvimento, em que os problemas de competitividade internacional são graves e associados às crises recorrentes no balanço de pagamentos, é importante que a regra monetária seja consistente com o equilíbrio em conta corrente. Finalmente, analisam-se as condições que garantem a estabilidade do sistema sob distintas regras monetárias. 
O artigo divide-se em quatro seções, além desta introdução e da conclusão. Na primeira seção é apresentada uma revisão de literatura a respeito do tema da política monetária sob o regime de metas de inflação, na tentativa de situar o leitor sobre as diferenças entre o chamado "novo consenso" e o pensamento póskeynesiano. Na segunda seção é apresentada a estrutura básica do modelo, a saber, a curva de Phillips derivada de uma economia em concorrência imperfeita e barganha salarial, a curva $I S$ em termos da taxa de utilização da capacidade instalada, a dinâmica da taxa de juros e a dinâmica da taxa de câmbio real. Na terceira seção são analisados o sistema dinâmico e as condições para a estabilidade do modelo. Finalmente, na quarta seção, são discutidas as implicações de política que surgem da dinâmica do modelo.

\section{Política monetária: o novo consenso e a critica pós-keynesiana}

Até pelo menos o final da década de sessenta o mainstream em Macroeconomia era o que se convencionou chamar de síntese neoclássica. Em linhas gerais, a síntese neoclássica foi a combinação das ideias de Hicks (1937), traduzidas no modelo IS-LM, com a relação empírica da curva de Phillips.

A síntese neoclássica sofreu muitos ataques teóricos que culminaram na revolução das expectativas racionais nos anos setenta. Desde então a Macroeconomia convencional caminhou por uma trajetória de debates sem que houvesse um consenso sobre a prática mais adequada das políticas macroeconômicas, uma vez que tanto ideias novo-clássicas como novokeynesianas faziam parte do mix possível de alternativas.

Em meados da década de noventa, um novo consenso ou nova síntese neoclássica começou a tomar forma (Arestis; Sawyer, 2003a; Goodfriend, 2004), incorporando de um lado ideias novo-keynesianas e de outro, arcabouços de modelagem influenciados pelos modelos de equilíbrio geral e pela incorporação de expectativas racionais. Nessa nova síntese neoclássica a forma majoritária de atuação da política macroeconômica é a política monetária, operacionalizada com auxilio de modelos intertemporais de equilíbrio geral (Arestis; Sawer, 2008; Woodford, 2004).

Na prática da política macroeconômica, a forma usual do novo consenso é a utilização do regime de metas de inflação. A adoção de regimes de metas implica no anúncio público de metas quantitativas para um índice de preços por parte da autoridade monetária. Essa meta deve ser o objetivo central da autoridade monetária que deve trabalhar para conseguir uma taxa de inflação baixa e estável. (Mishkin, 1999; Woodford, 2004).

Segundo Woodford (2004), a maior conquista do regime de metas foi o estabelecimento de alvos bem definidos para a política monetária, com alto grau de 
transparência e papel importante para projeções quantitativas da evolução futura da economia.

Há duas dimensões importantes a serem consideradas ao estudar o regime de metas dentro do escopo convencional, uma diz respeito a certos princípios que deveriam basilar a adoção de tal estratégia de política monetária. Mishkin (2000) aponta alguns destes princípios, a saber: 1) a estabilidade de preços promove benefícios substanciais; 2) a política fiscal deve ser alinhada com a política monetária; 3) a inconsistência dinâmica é um sério problema e deve ser evitada; 4) a política monetária deve olhar para frente (forward looking); 5) a prestação de contas é um princípio básico da democracia; 6) a política monetária deve se preocupar com as flutuações do produto e dos preços; 7) as recessões econômicas mais graves são associadas à instabilidade financeira.

A outra dimensão se relaciona com as bases teóricas da macroeconomia convencional contemporânea. Seguindo as considerações de Taylor (2000), as bases teóricas da macroeconômica convencional podem ser descritas em "cinco princípios": 1) o produto real de longo prazo é determinado pelo lado da oferta através de deslocamentos da função de produção; 2) não existe trade-off entre a inflação e desemprego no longo prazo; 3) existe trade-off entre inflação e desemprego no curto prazo; 4) a expectativa da inflação e das decisões políticas futura são endógenas e quantitativamente significantes; 5) a política monetária deve ser baseada numa função de reação em que a taxa nominal de juros de curto prazo deve ser o instrumento de política monetária.

\subsection{A critica pós-keynesiana}

A compreensão dos mecanismos fundamentais da macroeconomia diverge entre autores ligados ao novo consenso e à escola pós-keynesiana. Desta maneira, essas divergências fazem com que tanto o regime de metas como o arcabouço teórico do novo consenso (entendido de maneira mais ampla) esteja sujeito a criticas.

Arestis e Swayer (2006) e Lavoie (2004), apontam alguns dos principais problemas da estrutura básica da política monetária convencional: i) Os modelos convencionais se assentam na ideia de taxa natural de crescimento do produto unicamente determinado pelo lado da oferta. No pensamento pós-keynesiano o produto de longo prazo é dependente do estado da economia no curto prazo através de processos de path-dependence de maneira que não faz sentido pensar em "produto natural". ii) A ideia de "taxa natural de crescimento do produto" está consequentemente ligada à noção de neutralidade da moeda, especialmente no longo prazo. No pensamento pós-keynesiano a moeda é não neutra, mesmo no longo prazo. iii) No escopo do novo consenso, a inflação é um fenômeno de 
demanda. No pensamento pós-keynesiano, como aponta Davidson (1991), a ocorrência da inflação é o sintoma de uma luta entre os grupos organizados, cada um tentando obter uma parcela maior da renda [nacional ou mundial] disponível. Sendo assim, a devida compreensão do fenômeno inflacionário não pode negligenciar o conflito distributivo inerente às economias capitalistas, puxado de um lado pela margem de lucro desejada pelos capitalistas e, do outro, pelo salário desejado pelos trabalhadores. iv) No novo consenso advoga-se pela existência de uma taxa de juros natural à la Wicksell, de modo que a regra de política monetária deve considerar a taxa natural de juros na sua função de reação. Na tradição póskeynesiana, a taxa de juros é um fenômeno monetário, da mesma maneira que não faz sentido falar em taxa natural de crescimento, não faz sentido pensar em uma taxa natural de juros.

As criticas apresentadas acima podem levar à conclusão de que o regime de metas é completamente incompatível com o pensamento pós-keynesiano, como defendem, por exemplo, Arestis e Sawyer (2003a, 2003b), não obstante, autores como Setterfield (2006), Lima e Setterfield (2008) e Oreiro e Rocha (2008) têm advogado pela possibilidade do regime de metas ser compatível com o pensamento pós-keynesiano, desde que levadas em conta tais criticas na formulação da política monetária.

Segundo Setterfiled (2008), o regime de metas é compatível com o pensamento pós-keynesiano desde que: a) Seja considerado o fato de que a demanda agregada tem um papel importante na determinação da renda real; b) Leve-se em conta o papel do conflito distributivo (conflicting claims) na explicação do processo inflacionário. c) Seja dado menos ênfase a níveis muito baixos de inflação e maior ênfase a metas de produto e, consequentemente, nível de emprego.

A partir da próxima seção, é desenvolvido um modelo que busca levar em conta parte das críticas levantadas nessa seção, dando ênfase especial ao conflicting claims, ao papel da demanda agregada no médio/longo prazo e à importância de metas de produto/utilização da capacidade instalada.

\section{A estrutura básica do modelo}

O comportamento das firmas é dado pela conhecida equação de mark-up, consequência de uma economia em concorrência imperfeita.

$$
P=Z\left(\frac{W}{a}\right)
$$

Fazendo a diferenciação logarítmica da equação (1) obtém-se a dinâmica da inflação:

$$
p=\hat{z}+\widehat{W}-\hat{a}
$$


As variáveis com o símbolo “^” representam taxas de variação. Assume-se que o mark-up é constante no tempo e que a produtividade cresce a uma taxa exógena $\bar{a}$. Nesta economia simplificada, a demanda por salários nominais é o principal elemento da dinâmica dos preços - esta última, por sua vez, fruto de um processo de "conflito distributivo" semelhante ao apresentado por Rowthorn (1977). Na tradição pós-keynesiana, em última instância, o nível de emprego é uma decisão dos empresários. Aos trabalhadores resta demandar salários nominais e eles fazem isso com vistas a manter ou aumentar sua participação na renda nacional. Para manter sua participação na renda nacional, os trabalhadores procuram compensar as perdas inflacionárias, assim como se apropriar dos ganhos de produtividade. Um aspecto adicional a ser considerado, como se trata de uma economia aberta, é que os trabalhadores compram bens importados, e é plausível pensar que o câmbio real tem algum impacto sobre a demanda salarial.

Neste texto usa-se o suposto de que, dado um câmbio favorável, os trabalhadores aceitam menor participação na renda pensando na possibilidade de maior poder de compra relativamente aos bens importados. Existe, assim, certa compensação entre o câmbio real e a demanda de participação dos trabalhadores na renda. Na tradição pós-keynesiana, hipótese semelhante é utilizada por Blecker $(1999)^{1}$, na literatura convencional o artigo seminal de Svensson (2000) inclui a taxa de câmbio na curva de Phillips usando como argumento complementar o efeito da taxa de câmbio sobre o processo de fixação de salários.

Empiricamente, há uma vasta literatura sobre pass-through cambial, dos quais pode-se citar Calvo e Reinhart (2000), Goldfajn e Werlang (2000) e SchmidtHebbel e Tapia (2002). Nestes textos são mostradas evidências de que o passthrough cambial tende a ser maior em países emergentes do que em países desenvolvidos. Certamente por conta disso o canal cambial da política monetária é mais importante nos países da América Latina, como apontam Barbosa (2008) e Galindo e Ros (2008), do que o canal do crédito.

A existencia de pass-through é um bom argumento empírico para inclusão da taxa de câmbio na barganha salarial, contudo é importante questionar se existe algum argumento empírico que sugira a existência de impactos das taxas de câmbio diretamente sobre os salários. Dentre os artigos que investigam essa questão podese citar os textos de Revenga (1992), Campa e Goldberg (2001) e Bertrand (2004). Estes autores encontram, para os Estados Unidos, evidências de que a apreciação cambial (através dos ganhos de competitividade dos produtos estrangeiros) tem impacto negativo sobre os salários. Por sua vez, considerando mercados de trabalho sindicalizados, Bastos e Wright (2009) encontram, para economia

(1) É interessante observar que ela pode ser derivada também de um processo de barganha salarial baseado no salário eficiência, no qual os trabalhadores procuram manter seus ganhos reais em termos absolutos, e não apenas em relação aos outros trabalhadores. Ver Sorensen e Whitta-Jacobsen (2005). 
portuguesa, evidências de que a taxa de câmbio tem impacto sobre o processo de barganha salarial, sendo que as duas principais conclusões do texto são: i) Existe uma relação negativa entre apreciação cambial e salários. ii) Apreciações cambiais tendem a diminuir a sensibilidade dos salários à taxa local de desemprego.

Com base nas hipóteses apresentadas acima, segue equação que descreve a dinâmica dos salários nominais:

$$
\widehat{W}=p^{e}+\bar{a}+(1-\emptyset)\left(\omega^{d}-\omega^{f}\right)+\emptyset q \quad 0<\emptyset<1
$$

Nesta equação $p^{e}$ é a expectativa inflacionária, $\omega^{d}$ é a participação na renda desejada pelos trabalhadores, $\omega^{f}$ é a participação efetiva (definida pelo mark-up das firmas), $q$ é o câmbio real e $\emptyset$ é o parâmetro que define a ponderação entre demanda por participação na renda e desejo de poder de compra relativo ao câmbio. Note que, quanto maior for $\emptyset$, maior a importância dos bens importados na cesta de consumo dos trabalhadores, o que também pode ser interpretado como maior abertura comercial da economia.

Adota-se a hipótese de que a participação na renda desejada pelos trabalhadores é uma função positiva do nível de utilização da capacidade instalada $u$.

$$
\omega^{d}=\alpha u
$$

Combinando (2), (3) e (4) obtém-se a curva de Phillips Pós-Keynesiana:

$$
p=p^{e}+(1-\emptyset)\left(\omega^{d}-\omega^{f}\right)+\emptyset q
$$

Considera-se que as expectativas inflacionárias convergem para a meta de inflação adotada pelo banco central. Esta é basicamente a mesma hipótese utilizada por Lima e Setterfild (2008). Baseados nas ideias de Dequech (2000), estes autores mostram que a inflação pode ser escrita como uma expectativa condicional, isto é, $p^{e}=E(p \mid \Omega)$, onde $\Omega$ captura a influencia das informações disponíveis e do animal spirits dos empresários. Lima e Setterfield (2008) argumentam que a meta de inflação é um exemplo especifico de como as autoridades macroeconômicas podem se engajar na criação de "convenções" que ancorem as expectativas. Seguindo esse raciocínio, desde que os agentes considerem a política monetária crível, a expectativa inflacionária será ancorada pela meta, $E(p \mid \Omega)=\bar{p}$, de modo a fornecer a seguinte curva de Phillips:

$$
p=\bar{p}+(1-\emptyset)\left(\omega^{d}-\omega^{f}\right)+\emptyset q
$$

Além da justificativa teórica, a hipótese de convergência expectacional encontra subsidio em várias evidências empíricas. Desde as primeiras experiências de regimes de meta de inflação, no início dos anos noventa, trabalhos têm procurado avaliar a efetividade das metas de inflação e, em especial, a capacidade 
do regime de ancorar as expectativas. Para os países desenvolvidos a referência clássica é Bernanke et al (1999). Segundo estes autores, em países como Nova Zelândia, Canadá, Reino Unido e Suécia, o regime de metas tem tido êxito em ancorar as expectativas dos agentes. Para os países emergentes, Mello et al (2008) trazem uma série de trabalhos empíricos que incluem países como Brasil, Chile, República Checa, México, África do Sul e Turquia. A evidência apontada neste livro é de que, na maioria dos países emergentes cobertos na amostra, a meta tem ancorado as expectativas inflacionárias. No que diz respeito ao Brasil, Bevilaqua, Mesquita e Minella (2007) apresentam evidências empíricas de que o regime de metas também tem agido como importante atrator das expectativas.

\subsection{A demanda agregada e o equilíbrio no mercado de bens}

Parte-se de uma função de demanda agregada com diferenças nas propensões a poupar de empresários e trabalhadores.

$$
Y=c_{w}(1-\pi) Y+c_{k} \pi Y+I+B
$$

Como sugerido por Blecker (2010), as variáveis serão definidas como proporção do estoque de capital, na medida em que se busca encontrar uma curva IS relacionada com a capacidade instalada da economia, seguindo um procedimento semelhante à Marglin e Bhaduri (1990).

$$
\frac{Y}{K}=c_{w}(1-\pi)\left(\frac{Y}{K}\right)+c_{k} \pi\left(\frac{Y}{K}\right)+\frac{I}{K}+\frac{B}{K}
$$

Tem-se que $\bar{Y}$ é o produto potencial, $\frac{Y}{\bar{Y}}=u$ é a taxa de utilização da capacidade instalada da economia, $v=\frac{\bar{Y}}{K}$ é o inverso da relação capital produto (constante por simplicidade), $g$ é o investimento como proporção do estoque de capital $K$ e $h$ é a conta corrente como proporção do estoque de capital. Reescrevendo a equação (7):

$$
u v=c_{w}(1-\pi) u v+c_{k} \pi u v+g+h
$$

Assumem-se as seguintes funções lineares para o investimento e para a conta corrente:

$$
\begin{gathered}
g=\tau+\delta_{1} u-\delta_{2} r \\
h=\sigma+b_{1} q-b_{2} u
\end{gathered}
$$

Em que $q$ é a taxa de câmbio real. As funções da conta corrente são as convencionais - taxa de câmbio real e capacidade utilizada (que reflete variações no produto), no caso do comércio internacional, e taxa de juros e o acelerador, no caso do investimento. Reescrevendo a curva de demanda: 


$$
u v=c_{w}(1-\pi) u v+c_{k} \pi u v+\tau+\delta_{1} u-\delta_{2} r+\sigma+b_{1} q
$$

Por conveniência, escreve-se $c_{w}(1-\pi)+c_{k} \pi=c$, com $0<c<1$.

$$
u v=c u v+\tau+\delta_{1} u-\delta_{2} r+\sigma+b_{1} q-b_{2} u
$$

O equilíbrio no mercado de bens implica na seguinte $I S$ :

$$
\begin{gathered}
u v-c u v-\delta_{1} u+b_{2} u=\tau-\delta_{2} r+\sigma+b_{1} q \\
u\left[v(1-c)-\delta_{1}+b_{2}\right]=\alpha-\delta_{2} r+\sigma+b_{1} q \\
u=\frac{\tau-\delta_{2} r+\sigma+b_{1} q}{b_{2}-\delta_{1}+v s}
\end{gathered}
$$

$\mathrm{O}$ equilíbrio de curto prazo do modelo requer que a desigualdade $b_{2}+$ $v S>\delta_{1}$ seja verificada de modo a se obter uma curva $I S$ com inclinação negativa. Essa é uma hipótese conveniente sobre o formato da curva IS que também pode ser encontrada em Blecker (2010) no desenvolvimento de um modelo póskeynesiano/kaleckiano. Intuitivamente, a desigualdade acima significa que a soma da poupança interna e externa devem superar o impacto do aumento da renda/utilização da capacidade instalada sobre o investimento. Em outras palavras, toda vez que a demanda agregada/utilização da capacidade aumentar, o excesso de demanda será amortecido pela poupança interna e pelas importações, ao invés de exacerbado pelo aumento do investimento.

\subsection{A política monetária}

Desde o artigo seminal de Taylor (1993), a ideia de uma regra de taxa de juros como guia da política monetária tem sido utilizada tanto nas pesquisas acadêmicas, quanto nos modelos de boa parte dos bancos centrais ao redor do mundo, transformando-se em uma espécie de novo consenso. "The key assumption of the new approach is that the central bank follows a real interest rate rule; that is, it acts to make the real interest rate behave in a certain way as a function of macroeconomic variables such as inflation and output." (Romer, 2000, p. 6). Essa perspectiva coincide com a ideia defendida pelos economistas pós-keynesianos de que a curva de oferta de moeda de fato é endógena - a oferta de moeda responde passivamente à demanda a um certo preço, definido pela taxa de juros do banco central. Como indica Moore (2006), os bancos centrais não têm outra opção na operacionalização da política monetária, a não ser conduzir a taxa de juros da economia oferecendo liquidez sob a demanda do sistema bancário (Fontana; Setterfield, 2009). Contudo, nos modelos tradicionais de política monetária, a adoção de uma regra é algo que soa apenas como uma adequação técnica, fruto da impossibilidade prática do controle do agregado monetário. As hipóteses fundamentais que balizam a regra é a existência de uma taxa natural de 
crescimento do produto, a existência de uma taxa natural de juros e a neutralidade da moeda no longo prazo.

Em contraste com essas hipóteses, o paradigma pós-keynesiano enxerga a moeda como não-neutra no longo prazo, em que não existe taxa natural de crescimento do produto e muito menos taxa natural de juros. Não obstante, ainda sem esses balizadores exógenos é possível utilizar uma regra de taxa de juros. A razão para isso não é apenas prática, mas uma razão teórica. Como apontam Moore (2006) e Fontana e Setterfield (2009), em uma economia complexa, como a do capitalismo moderno, a base monetária e a oferta de moeda são completamente endógenas. A oferta de dinheiro de crédito é determinada pela demanda por crédito oriunda dos bancos, [sendo assim] as autoridades monetárias são incapazes de aumentar ou diminuir a oferta de moeda exógena a seu critério.

Segundo Setterfield (2006), seria possível compatibilizar uma regra de política monetária com metas de inflação dentro do paradigma pós-keynesiano, desde que se promovesse uma reestruturação nas linhas gerais destes modelos.

A primeira reestruturação necessária deve ocorrer na curva de Phillips da economia. Nos modelos deste novo consenso é utilizada uma curva de Phillips reduzida, na qual não se consideram explicitamente os salários, os custos dos bens intermediários e os preços dos importados (Arestis; Sawyer, 2006). No caso do modelo aqui desenvolvido, a derivação da curva de Phillips (tal como já foi apresentada na seção anterior), leva em conta de modo explícito o conflito distributivo, a barganha salarial e o preço dos importados, em uma economia que funciona em concorrência imperfeita ${ }^{2}$.

Quanto à política monetária, além de descartar a noção de taxa natural de crescimento do produto e taxa natural de juros, constrói-se um mecanismo no qual se inclui a taxa de utilização da capacidade instalada como um dos determinantes da taxa de juros reais. Formalmente, a regra monetária se exprime como:

$$
\dot{r}=\gamma(p-\bar{p})+\beta(u-\bar{u})
$$

Essa regra monetária leva em conta não apenas a meta de inflação $(\bar{p})$, mas também o efeito do aumento da capacidade utilizada sobre a decisão do governo de elevar os juros, tendo como referência uma meta para a taxa de utilização da capacidade instalada $(\bar{u})$.

(2) Apesar de reconhecer explicitamente o papel da taxa de câmbio real, é mantida, no trabalho, a hipótese simplificadora da ausência de custo dos bens intermediários. Ver também Blecker (2010). 


\subsection{A taxa de câmbio real}

A discussão anterior atribui um papel relevante à taxa de câmbio real na barganha salarial e no comportamento da demanda agregada. Por essa razão, é importante discutir a dinâmica da taxa de câmbio em mais detalhes.

Sabe-se que o câmbio real é definido como:

$$
q=\frac{P^{*} E}{P}
$$

E diferenciando logaritmicamente, é obtida a evolução dessa variável no tempo:

$$
\hat{q}=p^{*}+\hat{e}-p
$$

Assume-se que a economia do modelo é uma pequena economia aberta, com regime de câmbio flutuante e imperfeita mobilidade de capitais. As equações (15) e (16) descrevem a dinâmica do câmbio nominal ${ }^{3}$ e a dinâmica do câmbio real, respectivamente.

$$
\begin{array}{r}
\hat{e}=-\mu\left[\left(r+p^{e}\right)-\left(r^{*}+p^{* e}\right)\right], \quad \mu>0 \\
\hat{q}=(1+\mu) p^{*}-\mu\left(r-r^{*}\right)-\mu \bar{p}-p
\end{array}
$$

Sabe-se que a expectativa inflacionária $\left(p^{e}\right)$ converge para a meta de inflação $(\bar{p})$. Por sua vez, a expectativa de inflação do resto do mundo $\left(p^{*}\right)$ converge para a efetiva inflação do resto do mundo $\left(p^{*}\right)$ que, assim como a taxa de juros externa $\left(r^{*}\right)$, é exógena no modelo. No entanto, a taxa de juros interna é endógena, de tal forma que a equação (16) e a equação (13) formam um sistema de equações diferenciais cujas condições de estabilidade devem ser analisadas.

\section{0 sistema dinâmico}

Combinando de modo conveniente a curva de Phillips, a curva $I S$, a dinâmica da taxa de câmbio e a regra de política monetária, obtém-se o sistema de equação que segue:

$$
\dot{r}=A+L_{1} r+L_{2} q
$$

(3) Da paridade descoberta da taxa de juros nominais tem-se que:

$$
e^{e}{ }_{t+1}-e_{t}=i_{t}-i^{*}
$$

Assumindo que os agentes formam suas expectativas de modo regressivo e imaginam existir um nível "normal" de taxa de câmbio nominal, pode-se escrever a seguinte equação:

$$
e^{e}{ }_{t+1}-e_{t}=\theta\left(\overline{e_{t}}-e_{t}\right)
$$

Uma forma simplificada de descrever a taxa "normal" de câmbio é relacioná-la diretamente com a taxa de câmbio passada, $\overline{e_{t}}=e_{t-1}$, deste modo:

$\theta\left(e_{t-1}-e_{t}\right)=i_{t}-i^{*}{ }_{t}, \Delta e=-\theta^{-1}\left(i_{t}-i^{*}{ }_{t}\right), \quad \theta^{-1}=\mu$

Para uma discussão mais detalhada ver Sørensen e Whitta-Jacobsen (2005). 


$$
\hat{q}=B+L_{3} r+L_{4} q
$$

Em que:

$$
\begin{gathered}
A=\gamma\left[(1-\emptyset)\left(\alpha\left(\frac{\tau+\sigma}{b_{2}-\delta_{1}+v s}\right)-\omega^{f}\right)\right]+\beta\left(\frac{\tau+\sigma}{b_{2}-\delta_{1}+v s}-\bar{u}\right) \\
B=(1+\mu) p^{*}+\mu r^{*}-(1+\mu) \bar{p} \\
-(1-\emptyset)\left(\alpha\left(\frac{\tau+\sigma}{b_{2}-\delta_{1}+v s}\right)-\omega^{f}\right)
\end{gathered}
$$

$\mathrm{E}$ as derivadas parciais:

$$
\begin{aligned}
& \frac{\partial \dot{r}}{\partial r}=\frac{\gamma(\emptyset-1) \alpha \delta_{2}-\beta \delta_{2}}{b_{2}-\delta_{1}+v s}=L_{1} \\
& \frac{\partial \dot{r}}{\partial q}=\frac{\gamma(1-\emptyset) \alpha b_{1}+\beta b_{1}}{b_{2}-\delta_{1}+v s}+\gamma \emptyset=L_{2} \\
& \frac{\partial \widehat{q}}{\partial r}=-\mu+\frac{(1-\emptyset) \alpha \delta_{2}}{b_{2}-\delta_{1}+v s}=L_{3} \\
& \frac{\partial \widehat{q}}{\partial q}=\frac{(\emptyset-1) \alpha b_{1}}{b_{2}-\delta_{1}+v s}-\emptyset=L_{4}
\end{aligned}
$$

A matriz Jacobiana:

$$
J=\left[\begin{array}{ll}
L_{1} & L_{2} \\
L_{3} & L_{4}
\end{array}\right]
$$

O traço desta matriz é negativo, uma das condições para a estabilidade do sistema.

$$
L_{1}+L_{4}<0
$$

É preciso analisar o determinante:

$$
\begin{gathered}
L_{1} L_{4}-L_{2} L_{3}>0 \\
\left\{\frac{\gamma(\varnothing-1) \alpha \delta_{2}-\beta \delta_{2}}{b_{2}-\delta_{1}+v s}\right\}\left\{\frac{(1+\mu)(\emptyset-1) \alpha b_{1}}{b_{2}-\delta_{1}+v s}-(1+\mu) \emptyset\right\}=L_{1} L_{4} \\
\left\{\frac{\gamma(1-\emptyset) \alpha b_{1}+\beta b_{1}}{b_{2}-\delta_{1}+v s}+\gamma \emptyset\right\}\left\{-\mu+\frac{(1+\mu)(1-\emptyset) \alpha \delta_{2}}{b_{2}-\delta_{1}+v s}\right\}=L_{2} L_{3}
\end{gathered}
$$

O sistema dinâmico produz um equilíbrio estável quando a seguinte condição for satisfeita:

$$
\frac{(1-\emptyset) \alpha \delta_{2}}{b_{2}-\delta_{1}+v s}<\mu
$$


Tal condição pode ser alcançada em três casos:

1) Quando $\alpha$ é um parâmetro não muito grande, isto é, o impacto do produto sobre a barganha salarial é pequeno;

2) Quando o peso dos importados na cesta de consumo (e na barganha salarial) é alto, o que implica em um valor razoavelmente pequeno para $(1-\emptyset)$;

3) Finalmente, quando o impacto dos juros sobre o investimento é pequeno, isto é, quando $\delta_{2}$ for reduzido.

Sendo qualquer uma destas condições atendidas, têm-se as seguintes isóclinas e os respectivos valores de steady state:

\begin{tabular}{|c|c|c|c|}
\hline \multirow{2}{*}{$(22)$} & $\dot{r}=0$ & $r=\frac{A+L_{2} q}{-L_{1}}$ & $r^{*}=\frac{A-\left(L_{2} / L_{4}\right) B}{\left(\frac{L_{2} L_{3}}{L_{4}}-L_{1}\right)}$ \\
\hline$L_{1}<0, \quad L_{2}>0$ & $q^{*}=\frac{B-\left(L_{3} / L_{1}\right) A}{\left(\frac{L_{2} L_{3}}{L_{1}}-L_{4}\right)}$ \\
\hline$(23)$ & $\hat{q}=0$ & $r=\frac{B+L_{4} q}{-L_{3}}$ & \\
\hline
\end{tabular}

Figura 1

Diagrama de fases do modelo

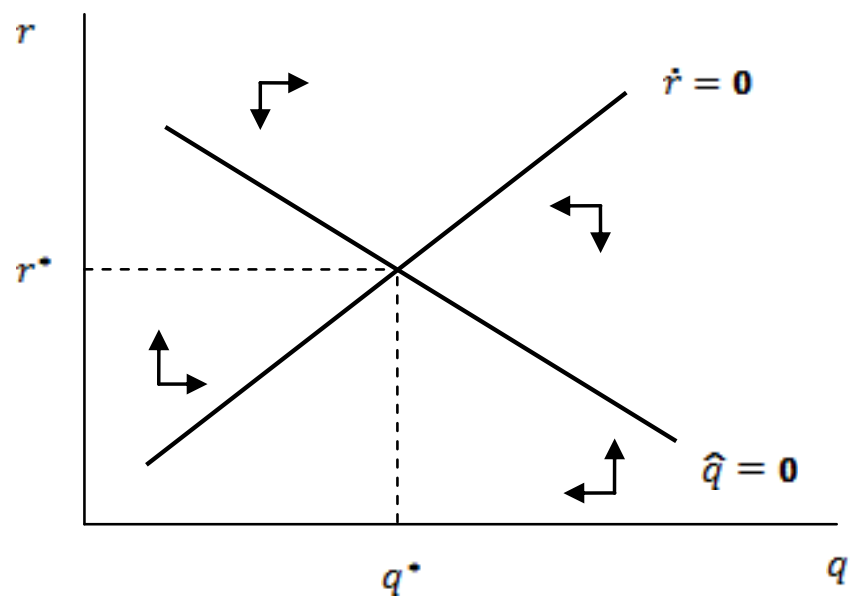

O diagrama de fase indica que o sistema é um nó estável, sob as condições antes definidas. Em equilíbrio, o Banco Central não terá motivos para alterar a taxa de juros. Como ao mesmo tempo os trabalhadores verão confirmadas suas expectativas de participação na renda, ajustada pela taxa de câmbio real, então o mercado de trabalho também se encontrará em equilíbrio. 


\section{Política macroeconômica e crescimento}

Verificada a estabilidade do modelo e partindo dos valores de equilíbrio das variáveis de estado, é possível realizar alguns exercícios de dinâmica comparativa para entender como diferentes formulações de política macroeconômica podem influenciar o crescimento econômico e a estabilidade da economia.

\subsection{Aumento da meta de inflação}

Para os valores de estado estacionários do câmbio e da taxa de juros, a taxa de crescimento da economia é a seguinte:

$$
g^{*}=\tau+\frac{\delta_{1}(\tau+\sigma)}{b_{2}-\delta_{1}+v s}-\left[\delta_{2}\left(1+\frac{\delta_{1}}{b_{2}-\delta_{1}+v s}\right)\right] r^{*}+\left[\frac{\delta_{1} b_{1}}{b_{2}-\delta_{1}+v s}\right] q^{*}
$$

Uma das formas de flexibilização da política monetária é admitir um aumento da meta de inflação. Os impactos desta possível mudança da política monetária sobre o crescimento podem ser apreendidos pela seguinte derivada parcial:

$$
\begin{gathered}
\frac{\partial g^{*}}{\partial \bar{p}}=-C \frac{\partial r^{*}}{\partial \bar{p}}+D \frac{\partial q^{*}}{\partial \bar{p}} \\
{\left[\delta_{2}\left(1+\frac{\delta_{1}}{b_{2}-\delta_{1}+v s}\right)\right]=C} \\
{\left[\frac{b_{1}}{b_{2}-\delta_{1}+v s}\right]=D} \\
\frac{\partial r^{*}}{\partial \bar{p}}=\frac{(1+\mu) \frac{L_{2}}{L_{4}}}{\left(\frac{L_{2} L_{3}}{L_{4}}-L_{1}\right)} \\
\frac{-(1+\mu)}{\left(\frac{L_{3} L_{2}}{L_{1}}-L_{4}\right)} \\
\frac{\partial g^{*}}{\partial \bar{p}}=(1+\mu)\left(\frac{-C L_{2}-L_{1} D}{L_{2} L_{3}-L_{1} L_{4}}\right)>0
\end{gathered}
$$

O modelo indica a existência de um trade-off de longo prazo entre inflação e crescimento, de modo que um aumento da meta de inflação pode ser usado como ferramenta de política. A existência de uma relação positiva entre crescimento e inflação remete à conclusões da literatura keynesiana dos anos sessenta, como se pode ver em Tobin (1965). O resultado mostrado na equação (22) é análogo ao 
conhecido efeito Mundell-Tobin ${ }^{4}$, isto é, a elevação da meta significa maior inflação, que gera reduções nas taxas de juros reais estimulando, assim, o investimento.

$\mathrm{Na}$ literatura pós-keynesiana recente, Setterfield (2006a), de modo semelhante ao resultado da equação (22), encontra uma relação positiva entre aumentos da meta de inflação e crescimento econômico, através de um modelo linear para a economia fechada.

Nota-se que, no modelo desenvolvido no texto, optou-se por formulá-lo em termos de equações lineares ${ }^{5}$. Essa estratégia de modelagem tem vantagens em termos de simplicidade e intuição dos resultados, mas precisa ser analisada dentro de suas limitações. Em especial, a relação positiva entre inflação e crescimento econômico, embora documentada empiricamente, não costuma ser linear. É coerente imaginar que, a partir de certo nível, a inflação se torne intolerável para os vários agentes econômicos, implicando em instabilidade das expectativas e gerando incerteza nas tomadas de decisão.

Existe uma literatura empírica que levanta indícios sobre um comportamento não-linear da relação entre inflação e crescimento. Haveria, assim, um ponto de inflexão a partir do qual a relação entre essas duas variáveis seria nula ou negativa. Sarel (1996) estima que este ponto de inflexão ocorra a partir de uma inflação anual de $8 \%$, já Khan e Senhadji (2000) estimam que este ponto esteja entre $1 \%$ e $3 \%$ anuais para as economias industrializadas e entre $7 \%$ e $11 \%$ para as economias em desenvolvimento. Vale salientar que o tema é um debate empírico em aberto e deve ser levado em conta na formulação das políticas monetárias.

\subsection{Forma alternativa de política monetária}

Pode-se argumentar que existem limites para o aumento da meta de inflação, isto é, sendo a meta de inflação uma variável que ancora as expectativas dos agentes, aumentos recorrentes da meta poderiam não ser desejáveis. Esses aumentos poderiam conspirar contra a estabilidade das expectativas ou ainda agravar a incerteza. Uma forma alternativa de flexibilizar a política seria operar mudanças na meta para a taxa de utilização da capacidade instalada, $\bar{u}$. Essa meta de utilização pode refletir um valor crítico a partir do qual se considera que aumentos da demanda são inflacionários, ou podem representar um objetivo de taxa de emprego ou de equilíbrio externo.

$$
\dot{r}=\gamma(p-\bar{p})+\beta(u-\bar{u})
$$

(4) Ver Tobin (1965) e Mundell (1963).

(5) A estratégia de modelagem linear é semelhante à adotada por Setterfield (2006a) e Lima e Setterfield (2008). Um interessante modelo não linear levando em conta o problema da inflação e o crescimento pode ser encontrado em Oreiro e Neves (2010), para o caso de uma economia fechada. 
O uso de uma meta como esta implica na existência de uma sensibilidade maior do police maker em relação a níveis baixos de utilização da capacidade instalada. Evidentemente, o efeito de um aumento na meta da taxa de utilização da capacidade instalada é positivo:

$$
\begin{gathered}
\frac{\partial g^{*}}{\partial \bar{u}}=-C \frac{\partial r^{*}}{\partial \bar{u}}+D \frac{\partial q^{*}}{\partial \bar{u}} \\
\frac{\partial r^{*}}{\partial \bar{u}}=\frac{-\beta L_{4}}{L_{2} L_{3}-L_{1} L_{4}} \\
\frac{\partial q^{*}}{\partial \bar{u}}=\frac{-\beta L_{1}}{L_{2} L_{3}-L_{4} L_{1}} \\
\frac{\partial g^{*}}{\partial \bar{u}}=\frac{C \beta L_{4}-D \beta L_{1}}{L_{2} L_{3}-L_{4} L_{1}}>0
\end{gathered}
$$

O uso de uma meta de capacidade instalada $\bar{u}$ não deveria ter um caráter arbitrário, mas estar associado a objetivos específicos da política, seja em termos do nível de emprego ou em termos de equilíbrio em conta corrente. A seguir analisar-se-á como o uso de uma meta de equilíbrio externo afeta o funcionamento do modelo. Acredita-se que este aspecto seja especialmente importante em economias como a brasileira (e nas economias latino-americanas em geral), onde são frequentes as crises geradas por um processo de excessivo endividamento nos mercados internacionais, em fases de ampla liquidez na economia mundial.

Da equação (10) pode ser obtida a taxa de utilização compatível com o equilíbrio em conta corrente:

$$
u^{E}=\frac{\sigma+b_{1} q}{b_{2}}
$$

Em se tratando de uma economia com fluxos de capitais, no curto prazo, os déficits em conta corrente podem ser financiados com poupança externa, o que em tese contornaria possíveis problemas. Como mencionado, ainda assim, parece plausível pensar que déficits recorrentes não sejam desejáveis, tendo efeitos deletérios sobre a trajetória de crescimento da economia.

Com a utilização da meta de capacidade compatível com o equilíbrio externo, surge um novo sistema de equações diferenciais no qual a seguinte equação substitui a equação (13):

$$
\frac{\partial \dot{r}}{\partial q}=\frac{\gamma(1-\emptyset) \alpha b_{1}+\beta b_{1}}{b_{2}-\delta_{1}+v s}+\gamma \emptyset-\frac{\beta b_{1}}{b_{2}}=L_{2}{ }^{*}
$$

O novo sistema só possui uma derivada parcial distinta daquele já apresentado. O novo Jacobaiano é dado por: 


$$
J=\left[\begin{array}{cc}
L_{1} & L_{2}^{*} \\
L_{3} & L_{4}
\end{array}\right]
$$

O traço de $J$ é negativo e o determinante de $J$ é positivo.

$$
\begin{gathered}
L_{1}-L_{4}<0 \\
L_{1} L_{4}-L_{2}{ }^{*} L_{3}>0
\end{gathered}
$$

As condições de estabilidade são as mesmas que já foram discutidas no sistema anterior.

O uso de uma regra de política monetária incluindo metas para o equilíbrio externo é desejável na medida em que garante que no longo prazo o sistema não estará acumulando um estoque excessivo de dívidas com o exterior, contudo, tal procedimento pode implicar em uma situação bastante restritiva. Do ponto de vista teórico, modelos de crescimento pós-keynesianos, dos quais se destacam os modelos desenvolvidos por Thirlwall (1979) e McCombie e Thirlwall (1994), vêm o Balanço de Pagamentos como a principal restrição para o crescimento de longo prazo. Em uma situação na qual a elasticidade-renda das importações seja mais alta que a elasticidade renda das exportações, uma meta de conta corrente do tipo apresentado aqui implicará em baixo crescimento econômico, uma vez que o nível da capacidade instalada compatível com o equilíbrio externo pode ser, nestes casos, extremamente baixo.

Há de se observar, portanto, que as condições de competição externa da economia (refletidas no parâmetro $\sigma$ ) possuem papel importante no que diz respeito às taxas de crescimento da economia.

$$
g=g\left(u^{E}(\sigma)\right), \quad g^{\prime}(\sigma)>0
$$

$\mathrm{Na}$ tentativa de suplantar as restrições externas ao crescimento, a estratégia de política monetária não deve ocorrer dissociada de objetivos de longo prazo relativos à estrutura competitiva da economia. É preciso reforçar a necessidade de, no longo prazo, a economia sofrer modificações tecnológicas que permitam equilíbrio externo com maior nível de emprego e maior crescimento.

\section{Conclusão}

Ao longo do trabalho foi proposto um modelo dinâmico em que o efeito das metas de inflação é analisado no contexto de uma perspectiva pós-keynesiana. Ao contrário dos modelos convencionais, o modelo aqui apresentado admite a existência de um trade-off de longo prazo entre inflação e crescimento, de modo que a flexibilização da política monetária, através de aumentos na meta de inflação, é também uma opção de política pró-crescimento. Assim, em concordância com a 
tradição pós-keynesiana, no modelo proposto, a política monetária tem efeitos reais.

Todavia, o grau de flexibilidade da meta de inflação é limitado. Na medida em que ela representa uma âncora para as expectativas dos agentes, se essa âncora muda com frequência (ou se é fixada em níveis altos demais em relação àquele para o qual as firmas e trabalhadores consideram que existe um compromisso razoável com a estabilidade), então existe a possibilidade de surgirem problemas sérios de incerteza crescente. Como alternativa, discute-se a possibilidade de incluir na regra monetária uma meta para o nível de capacidade utilizada. $\mathrm{O}$ aumento da meta de capacidade utilizada (supondo que existe uma margem para sua flexibilização) favorece o crescimento sem comprometer a estabilidade. Essa meta, no entanto, não é arbitrária e deveria estar associada a objetivos de combate ao desemprego ou refletir uma preocupação com a estabilidade externa da economia.

Com efeito, o tema do equilíbrio externo é um aspecto extremamente importante do desempenho das economias em desenvolvimento, muitas vezes negligenciado na abordagem convencional. É chave levar em conta a estabilidade do estoque de dívida externa e os impactos da política de metas de inflação sobre essa dívida. Brasil e América Latina mostram um histórico de crises externas que justificam plenamente essa preocupação. Por esse motivo, discute-se a inclusão de uma meta para a taxa de utilização da capacidade instalada em nível compatível com o equilíbrio em conta corrente. Tal inclusão não compromete a estabilidade do modelo.

Finalmente, é crucial levar em conta o fato de que o uso de metas para a conta corrente pode implicar em restrição ao crescimento econômico, como sugerem os modelos de crescimento à la Thirlwall. Nesse sentido, os resultados do trabalho reforçam a necessidade de associar a política monetária à políticas que levem em conta a competitividade externa, tais quais políticas industriais que objetivem mudar os parâmetros de competitividade de maneira a aproximar $u^{E}$ ao $u$ de pleno emprego em equilíbrio.

\section{Referências bibliográficas}

ARESTIS, P.; SAWYER, M. Inflation targeting: a critical appraisal. The Levy Economics Institute of Bard College, Sept. 2003a. (Working Paper).

The effectiveness of monetary policy and fiscal policy. Levy Economics Institute, 2003b. (Working Paper, n. 369).

; __ New consensus macroeconomics and inflation targeting: Keynesian

critique. Economia e Sociedade, Campinas, v. 17, Número especial, p. 631-655, dez. 2008. 
ARESTIS, P.; SAWYER, M. Monetary policy when money is endogenous. In: COMPLEXITY, endogenous money and macroeconomic theory: essays in honour of Basil J. Moore. 2006. p. 221-235.

BARBOSA, N. Inflation targeting in Brazil: 1999-2006. In: EPSTEIN, G.; YELDAN, E. (Ed.). Beyond inflation targeting: Central Bank policy for employment creation, poverty reduction and sustainable growth. Edward Elgar, 2008.

BASTOS P.; WRIGHT P. Exchange rates and wages in unionised labour markets. University of Nottingham, GEP, 2009. (Discussion Papers, 10/15).

BEVILAQUA, A. S.; MESQUITA M.; MINELlA, A. Brazil: taming inflation expectations. Central Bank of Brazil, Research Department, 2007. (Working Papers Series, n. 129).

BERNANKE, B. et al. Inflation targeting: lessons from the international experience. Princeton, NJ: Princeton University Press, 1999.

BERTRAND, M. From the invisible handshake to the invisible hand? How import competition changes the employment relationship. Journal of Labor Economics, v. 22, n. 4, 2004

BLECKER, R. A. Kaleckian macro models for open economies. In: DEPREZ, J.; HARVEY, J. T. (Ed.). Foundations of international economics: post Keynesian perspectives. London: Routledge, 1999. p. 116-149.

Open economy models of distribution and growth. Paper to be presented at the Eastern Economic Association annual meeting, Philadelphia, PA, Feb. 28, 2010.

CAMPA, J. M.; GOLDBERG L. S. Employment versus wage adjustment and the U.S.dollar. The Review of Economics and Statistics, v. 83, n. 3, 2001.

CALVO, G.; REINHART, C. Fixing for you life. NBER, 2000. (Working Paper).

DAVIDSON, P. Controversies in post Keynesian economics. London: Edward Elgar, 1991.

DEQUECH, D. Asset choice, liquidity preference, and rationality under uncertainty. Journal of Economic Issues, 54, p. 159-76, 2000.

FONTANA G.; SETTERFIELD M. Macroeconomics, endogenous money and contemporary financial crisis: a teaching model. J. Pluralism and Economics Education, vl. 1, n.. 1/2, p. 130-147, 2009.

GALINDO, L. M.; ROS, J. Inflation targeting Mexico: an empirical appraisal. International Review of Applied Economics, Mar. 2008.

GOLDFAJN, I.; WERLANG, S. The pass-through from depreciation to inflation: a panel study. Central Bank of Brazil, 2000. (Working Paper, n. 5).

GOODFRIEND, M. Monetary policy in the new neoclassical synthesis: a primer. Economic Quartely, Federal Reserve Bank of Richmond, v. 90/3, summer, 2004. 
HICKS, J. Mr. Keynes and the classics: a suggested interpretation. Econometrica, Apr. 1937.

KHAN, M. S.; SENHADJI, A. S. Threshold effects in the relationship between inflation and growth. IMF, Jun. 2000. (Working Paper).

LAVOIE, M. The new consensus on monetary policy seen from a post-Keynesian perspective. In: LAVOIE, M.; SECCARECCIA, M. (Ed.). Central banking in the modern world: alternative perspectives. Cheltenham, UK: Edward Elgar, 2004. p. 15-34.

LIMA, G. T.; SETTERFIELD, M. Inflation targeting and macroeconomic stability in a post Keynesian economy. Journal of Post Keynesian Economics, v. 30, p. 435-461, 2008.

MCCOMBIE, J. S. L.; THIRLWALL A. P. Economic growth and the balance of payments constraint. New York: St. Martin's Press, 1994.

MARGLIN, S. A.; BHADURI, A. Profit squeeze and Keynesian theory. In: MARGLIN, S. A.; SCHOR, J. B. (Ed.). The golden age of capitalism. Oxford University Press, 1990.

MELLO, L. de et al. Monetary policies and inflation targeting in emerging economies. OCDE, 2008.

MISHKIN, F. International experiences with different monetary policy regimes. NBER, Feb. 1999. (Working Paper, 6965).

What should central banks do? Federal Reserve Bank of St. Louis Review, v. 82 , n. 6, p. 1-13, 2000.

MOORE, B. J. Shaking the invisible hand: complexity, endogenous money and exogenous interest rates. Basingstoke: Palgrave Macmillan, 2006.

MUNDELL, R. Inflation and real interest. Journal of Political Economy, v. 71, n. 3, 1963.

OREIRO, J. L. C.; ROCHA, M. A. A experiência internacional de regimes de metas de inflação: uma análise de painel dinâmico. Nova Economia (UFMG), v. 18, p. 267-291, 2008 .

; NEVES, A. L. Acumulação de capital, utilização da capacidade produtiva e inflação. Uma análise a partir de um modelo pós-keynesiano não linear. Economia $e$ Sociedade, Campinas, v. 19, n. 1 (38), abr. 2010.

REVENGA, A. L. Exporting jobs? the impact of import competition on employment and wages in U.S. manufacturing. The Quarterly Journal of Economics, v. 107, n. 1, 1992.

ROMER, D. Keynesian macroeconomics without the LM curve. Journal of Economic Perspectives, v. 14, n. 2, p. 149-169, 2000.

ROWTHORN, R. Conflict, inflation and money. Cambridge Journal of Economics, 1, p. 215-239, 1977.

SAREL, M. Nonlinear effects of inflation on economic growth. IMF Staff Papers, v. 43, n. 1, p. 199-215, 1996. 
SETTERFIELD, M. Is inflation targeting compatible with post Keynesian economics? Journal of Post Keynesian Economics, 28, p. 653-671, 2006.

SCHMIDT-HEBBEL, K.; TAPIA, M. Inflation targeting in Chile. The North American Journal of Economics and Finance, v. 13, p. 125-146, 2002.

SORENSEN, P. B.;WHITTA-JACOBSEN, H. J. Introducing advanced macroeconomics: growth and business cycles. New York: McGraw-Hill, 2005.

SVENSSON, L. E. O. Open-economy inflation targeting. Journal of International Economics, v. 50, n. 1, p. 155-183, Feb. 2000.

TAYLOR, J. B. Discretion versus policy rules in practice. Carnegie-Rochester Conference Series on Public Policy, p. 195-214, 1993.

. Teaching modern macroeconomics at the principles level. The American Economic Review, v. 90, n. 2, p. 90-94, May 2000.

THIRLWALL, A. P. The balance of payments constraint as an explanation of international growth rates differences. Banca Nazionale del Lavoro Quarterly Review, 128, p. 45-53, 1979.

TOBIN, J. Money and economic growth. Econometrica, v. 33, n. 4, p. 671-684, 1965.

WOODFORD, Michael. Inflation targeting and optimal monetary policy. Federal Reserve Bank of St. Louis Review, v. 86, n. 4, p. 15-41, Jul./Aug. 2004. 